\title{
Infection Prevention Strategy in General Hospital under Regular Epidemic Prevention and Control
}

\author{
Yuanli Chen*, Ying Xue, Yingying Zhang, Huijun Zhou, Liang Peng \\ The Third Affiliated Hospital of Sun Yat-sen University, Guangzhou, China \\ Email: *cyl.ff@163.com
}

How to cite this paper: Chen, Y.L., Xue, Y., Zhang, Y.Y., Zhou, H.J. and Peng, L. (2021) Infection Prevention Strategy in General Hospital under Regular Epidemic Prevention and Control. Open Journal of Preventive Medicine, 11, 159-167. https://doi.org/10.4236/ojpm.2021.114013

Received: March 18, 2021

Accepted: April 27, 2021

Published: April 30, 2021

Copyright $\odot 2021$ by author(s) and Scientific Research Publishing Inc. This work is licensed under the Creative Commons Attribution International License (CC BY 4.0).

http://creativecommons.org/licenses/by/4.0/ (c) (i) Open Access

\begin{abstract}
New coronavirus pneumonia spread rapidly all over the world in the first half of 2020, which is a new respiratory disease with strong infectiousness. At present, the epidemic situation in China has been effectively controlled, but the global epidemic situation remains grim, and the National Health Commission has instructed that the prevention and control of new coronavirus pneumonia should become normalized. As outposts for surveillance of public health events, hospitals are an important front in the fight against the epidemic. Therefore, it is very crucial to construct infection prevention and control system actively to contain the outbreak. This paper analyzes and summarizes the normal prevention and control management strategy of Infection Control Branch Management (hereinafter referred to as Hospital Infection-Control Dept) in a certain designated comprehensive third-grade hospital in Guangdong Province and transforms the prevention and control means in emergency state into measures for sustainable development, which also provides basis and reference for Hospital Infection-Control Dept of medical institutions to formulate prevention and control guidelines.
\end{abstract}

\section{Keywords}

Normal Prevention and Control, Hospital Infection Management, Infection Prevention Strategy

\section{Introduction}

\subsection{Epidemic Situation}

Novel coronavirus pneumonia (hereinafter referred to as COVID-19) is a serious respiratory infectious disease caused by acute respiratory virus (SARS-CoV-2), which was included in category $B$ infectious diseases and managed in accordance 
with category A infectious disease in China [1]. People are generally susceptible [2]. "COVID-19 may become a long-term epidemic nationwide or locally", the Chinese Center for Disease Control and Prevention said in its latest CDC weekly report. At present, the epidemic situation of COVID-19 in China has not been completely contained in the prevention and control of the spread of overseas input and diffusion, and there are still aggregation cases in individual areas, and the epidemic situation is still uncertain.

\subsection{General Hospital Situation}

Hospitals have the character of big population density and strong fluidity, which is prone to nosocomial infection. In 2020, the number of outpatient and emergency visits in our hospital reached 3.5 million person-times. During the epidemic prevention and control period, guidelines on prevention and control work formulated by Hospital Infection-Control Dept ensured the scientific, standardized and standardized operation of the hospital.

\section{Responsibilities of the Hospital Infection-Control Dept: Work Content and Role in Epidemic Prevention and Control}

\subsection{The Work Responsibilities of the Hospital Infection-Control Dept}

The Hospital Infection-Control Dept should draw up the rules and regulations of infection management in all departments of the hospital, be responsible for monitoring the incidence of nosocomial infection, and actively carring out the training and examination of the knowledge and skills of prevention and control of nosocomial infection and occupational protection knowledge, skills training and assessment, etc.

\subsection{The Main Contents of Hospital Infection Prevention and Control}

Covering the "five each", that is, each person in the hospital, each item in the hospital, the layout of each area in the hospital, each work process in the hospital, the details of each work in the hospital [3].

\subsection{Give Full Play to the Role of the Hospital Infection-Control Dept during the Epidemic Prevention and Control Period}

According to COVID-19 prevention and control management documents issued by the State Council and Guangdong Province, the relevant procedures are formulated. Including: hospital prevention and control work system, protection training and assessment of all staff at all levels, emergency treatment drills for suspicious and confirmed cases, prevention and control work guidelines for clinical departments, prevention and control procedures for diagnosis and treatment operations in key departments, sewage treatment and environmental terminal disinfection, etc. The documents are sent to each department and section, which guide 
the staff to carry out scientific and reasonable protection and reduce the hidden danger of cross-infection. Secondly, the staff of the Hospital Infection-Control Dept should enter the site for supervision and inspection at any time, go deep into the clinical front-line departments and each section, actively find out problems and hidden dangers, and put forward professional opinions on the spot, supervise the rectification and follow-up tracking. The key points of supervision include: cleaning and disinfection of ward environment, personal protection of staff, transfer of medical waste, centralized management of inpatients, etc.

\section{Combination of Normalization Management and Emergency Prevention and Control}

\subsection{Normal Prevention and Control}

Formulate and improve the rules and regulations, the Hospital Infection-Control Dept assists to make fever treatment process and disinfection and isolation system and infectious disease reporting system. Standardize medical behavior with process and system and supervise and inspect it. Set up the position of supervisor of sense control, and act as the eagle-eye guardian of hospital sense control. Take epidemic prevention and control as an important part of hospital management and control, guide and supervise regularly, and conscientiously implement rules and regulations.

\subsection{Emergency Prevention and Control}

Start the emergency command strategy immediately. Relevant research shows that building a scientific and effective organization and command system can deal with public health emergencies more scientifically, effectively and timely [4]. To establish an emergency prevention and control organization system under the unified leadership and command of the Party Committee of the hospital, the members of the emergency prevention and control organization involve the leadership of the hospital, the competent authorities, the relevant key departments of the leadership and experts. Set up different levels of management system, including Prevention and control leading group, epidemic prevention monitoring group, medical treatment group, comprehensive security group, etc. It mainly makes overall arrangements for the following aspects: epidemic monitoring, personnel management, material supply, preview and triage, consultation of suspicious cases, management of inpatients, personal protection training, and disinfection of environment and articles. Hold regular meetings to find and solve problems early.

\section{Specific Measures for Prevention and Control of the Hospital Infection-Control Dept}

\subsection{Personnel Management}

\subsubsection{Training and Assessment of Staff of Various Departments in the Hospital}

The staff in each post in the hospital need to be trained on the knowledge of 
COVID-19 and the Hospital Infection-Control Dept organizes on-site protection training for different groups in the whole hospital, which is not only convenient for the staff of the hospital to learn but also avoids the risk of infection caused by the gathering of people during the epidemic. In high-risk departments, such as fever clinic and isolation ward, medical staff conduct one-to-one assessment in the aspects of preview and triage, identification of suspicious patients, ward management, personal protection, environmental cleaning and disinfection, etc., and they can only take up their posts after passing the assessment. Practice has proved that strengthening medical staff's awareness of protection and improving their ability to cope with COVID-19 and their sense of job responsibility are important measures to control the epidemic [5].

\subsubsection{Reasonable Working System of Medical Staff}

Combining with the reality of the hospital, we should rationally allocate human resources and shift arrangement, form a reasonable echelon, to ensure that the medical staff gets adequate rest. Secondly, pay attention to the health of medical staff and carry out health monitoring, including body temperature and respiratory system. In addition, talking with medical staff in different forms is also available to reduce their psychological anxiety [6].

\subsection{Strengthen the Management of Protective Items}

\subsubsection{Reasonable Distribution of Protective Equipment}

The Material supply section should ensure that the protective articles of the frontline departments are fully reserved. In the front-line departments of the hospital, such as dressing room and buffer zone of isolation ward and fever clinic, the schematic diagram of wearing and taking off protective clothing is posted, and the sense control supervisor is set up to supervise. Wearing and taking off personal protective equipment in strict accordance with the standard process can reduce the infection rate [7], and make the medical staff achieve zero infection during the epidemic.

\subsubsection{Disinfection Management of Goods and Environment}

According to the requirements of prevention and control of COVID-19, combined with the actual situation of the hospital, "COVID-19 Disinfection and Isolation Guide" is formulated. The consulting room, treatment room and observation room are disinfected with ultraviolet lamp for more than 1 hour when there is no one. In addition, the fever clinic keeps the air disinfection machine open continuously. $1000 \mathrm{mg} / \mathrm{L}$ chlorine-containing disinfectant is used to wipe and disinfect the surfaces of triage tables, hand washing basins and other objects four times a day. Ward bed units should be cleaned, disinfected and replaced regularly. Instruments and articles that need to be reused in isolated area should be marked, and sealed in double-layer special bags and sent to the Disinfection Supply Center for disinfection and sterilization, with high-pressure steam sterilization as the first choice. 


\subsection{Strengthen the Management of Outpatient and Emergency Department}

\subsubsection{Strictly Implement the Three-Level Screening System}

The first level screening is set at the entrance of the hospital, and all the admission personnel is required to wear medical-surgical masks, measure their body temperature and check their health codes. The second level screening is set at the triage table of each clinic department, and nurses at the triage table carry out temperature measurement and epidemiological history inquiry and registration. The three-level screening is set in the consultation room, and the doctor will register the epidemiological screening. If the body temperature is $\geq 37.3^{\circ} \mathrm{C}$ or the epidemiological history is abnormal, a special person will guide him to the fever clinic. Even after the patient leaves, the reception desk and the consultation room are disinfected. Secondly, both outpatient and emergency departments and inpatient departments implement the dual-channel system to further avoid cross-infection between patients and medical staff and reduce the risk of infection $[8]$.

\subsubsection{Management of Fever Clinic}

The fever clinic is set up with "three areas and two channels", and the clean area, potential pollution area and pollution area are clearly divided. Fully enclosed management is adopted internally, such as treatment process, registration, charging, laboratory tests and drug taking, etc. All of the above are done in the same area to avoid cross infection [9].

Standardized walking route management can make the shortest diagnosis and treatment path, complete the diagnosis and treatment activities in the region efficiently and safely, and also reduce cross-infection among patients. Medical staff should be treated with secondary protection when receiving patients, and register the epidemiological history of patients in detail. If suspicious cases appear, they should be immediately reported to the Medical Department and the Hospital Infection-Control Dept, and ask the expert group of COVID-19 for consultation. Patients will be sent to the isolation ward, which are also strictly forbidden to leave the hospital without permission.

\subsection{Strengthen the Infection Prevention and Control Management of General Inpatients}

\subsubsection{Admission Screening and Establishment of Buffer Ward}

All hospitalized patients have to go through three epidemiological screenings, namely, the entrance of the hospital, the inpatient department and the competent doctor, and they must carry the negative certificate of COVID-19 nucleic acid test ( 7 days before admission in low-risk areas, 3 days before admission in medium-risk areas, and the high-risk day) before admission. 2 - 3 buffer rooms are set up in each ward for temporary isolation of suspected cases of COVID-19 in hospitalized patients. 


\subsubsection{Establish Epidemic Reporting Guarantee Process and Emergency Plan}

Hospitals should routinely train and reserve emergency medical personnel to form a good emergency personnel scheduling system [10] and prepare for dispatching at any time. Based on the original infectious disease prevention and control system of the hospital, the Hospital Infection-Control Dept and the expert group of epidemic prevention and control update and improve emergency procedures and emergency plans, including procedures for treatment of suspected cases, procedures for inspection of suspected cases, procedures for diagnosis and treatment process of confirmed cases [11] and the emergency treatment process for medical staff after occupational exposure.

\subsubsection{Management of Accompanying and Visitation System}

Establish and improve the management system of accompanying and visitation. The ward is under 24-hour closed management, and all kinds of foreign personnel are not allowed to enter and leave the ward at will. Under special circumstances (such as change in patients' condition, need of diagnosis and treatment operation, admission and discharging, etc.), it is really necessary to enter the ward, check the health code (green code or blue code), measure the body temperature, wear a mask, fill in and sign the escort informed commitment. Inpatients must promise that only one companion can be left. During hospitalization, he shall abide by the hospital management system and consciously cooperate with the implementation of the system. Companions cannot change at will, who must consciously wear a mask, take body temperature 2 times a day and cannot go out at will. Accompanying and visitation is managed by "one patient, one companion and one wristband". Each patient can be visited only by 1 - 2 people at a time, and the visiting time of each person should not exceed 30 minutes. The ward shall register the information of visiting personnel and indicate the entry and exit time points (accurate to minutes). Doctors order to keep a companion and limit one companion per bed. Multiple attendants or taking turns accompanying is forbidden. Wrist bands are issued as companion certificate, including bed number, companion's name and companion's ID number to ensure that the companion and wrist band are consistent. The wrist bands of companion and patient are obviously different in color, with wrist bands of patients being blue and wrist bands of companions being yellow. In case of special circumstances, the new companion must complete the nucleic acid test before handling the replacement procedures and the old companion wrist band will be recovered and destroyed by the ward. Instruct companions to do personal protection. Entering and leaving the workplace of medical staff at will and visiting or gathering to chat is not acceptable.

\subsubsection{Infection Control and Management of Handymen in Hospital}

Handymen are part of the hospital. They spend most of their time in the hospit$\mathrm{al}$, and they are responsible for many links that come into contact with patients and pollutants, such as sanitary cleaning, transporting patients, transporting spe- 
cimens, collecting and transporting medical wastes, so the prevention and control management of these people is equally important. At present, hospital handymen are managed by outsourcing, so in the management, the clinical department and the outsourcing unit also have the responsibility of training, examination, supervision and management. The clinical department and the outsourcing unit jointly formulate the examination system and plan for the protection training of the workers, and carry out the training and examination on the basic knowledge of hospital prevention and control, hand hygiene, the use of surgical masks and protective equipment, the configuration of disinfectant, the disposal of medical wastes, etc. Only when the examination is passed can the workers take up their posts and ensure that the workers receive effective training. Infection control practitioners have the responsibility to supervise them.

\subsection{Application of Artificial Intelligence in Management}

\subsubsection{Application of Intelligent Disinfection Robot}

It adopts three modes of ultraviolet disinfection, hydrogen peroxide dry fog and air purification to disinfect the surface and air automatically or manually. It has the functions of autonomous navigation technology and avoiding objects in the environment. The remote control function of intelligent disinfection robot can reduce the exposure risk of workers and avoid the harm of ultraviolet rays and disinfectant to human body [12]. At the same time, we also purchased a number of other machines with various disinfection functions, which plays a certain role in disinfection management of nosocomial infection prevention and control.

\subsubsection{Application of Intelligent Cloud Monitoring System}

With the newly established isolation negative pressure ward, putting on and taking off protective clothing is an essential operation for us, and also a skill that medical workers must master. The newly introduced intelligent cloud monitoring system in our hospital has the functions of monitoring, supervision and voice guidance when workers are putting on and taking off the protective clothing, which improves the correct rate of putting on and taking off the protective clothing and reduces the risk of infection of the workers to some extent.

\section{Hospital Infection Prevention and Control Achievements}

By the end of December 2020, the number of fever clinics visits in our hospital had reached nearly 31 thousand, 155 patients were treated in isolation ward (Including 21 confirmed cases of COVID-19), and the staff of the whole hospital had no infection.

\section{Conclusion}

Since the end of 2019, COVID-19 has been in a worldwide pandemic for more than one year, with a cumulative total of over 115.7 million cases worldwide and over 2.5 million deaths [13]. China is still facing a high risk of imported cases; therefore, prevention and control should not be relaxed under normal circums- 
tances. "External defense input, internal defense rebound" has become the focus of epidemic prevention at present. Hospitals must pay attention to the prevention and control of infectious diseases and take scientific and effective measures to maintain human health [14]. According to relevant reports, there were a large number of cross-infections in hospitals in the early stage of COVID-19 [15]. Therefore, the prevention and control of the Hospital Infection-Control Dept is still a common phenomenon in medical institutions in the next period of time. All medical institutions should actively take scientific and effective preventive measures in the prevention and control of hospital sensation, prepare for a protracted war, especially in the aspects of strengthening the responsibility of Hospital Infection-Control Dept specialist, strengthening the function of fever clinic, management of outpatient and emergency department, and management of inpatient escort, etc. At the same time, do a good job in personal protection and environmental cleaning and disinfection of all personnel, standardize the prevention and control processes of various medical treatments and ensure the normal operation of various medical treatments in medical institutions. At the same time, hospitals should make full use of artificial intelligence to develop information technologies such as intelligent cloud monitoring, unmanned distribution logistics, intelligent robot disinfection, etc., so as to reduce the increased risk of infection due to personnel contact in hospitals. The practice has proved that in the sudden epidemic situation, the application of artificial intelligence has achieved maximum emergency prevention and control, which has certain promotion value for the normalization of prevention and control and even the future hospital service work.

\section{Acknowledgements}

This study was supported by grants from Tackling of key scientific and emergency special program of Sun Yat-sen University (SYSU-TKSESP) and emergency special program for 2019-nCoV of Guangdong province science and technology project (GDSTP-ESP) (2020B111105001).

\section{Conflicts of Interest}

The authors declare no conflicts of interest regarding the publication of this paper.

\section{References}

[1] National Health Commission of the People's Republic of China (2020) Announcement of the National Health Committee of the People's Republic of China (No.1 of 2020).

http://www.nhc.gov.cn/jkj/s7916/202001/44a3b8245e8049d2837a4f27529cd386.sht $\underline{\mathrm{ml}}$

[2] Guan, W.J., Chen, R.C. and Zhong, N.S. (2020) Strategies for the Prevention and Management of Coronavirus Disease 2019. European Respiratory Journal, 55, Article No. 2000597. https://doi.org/10.1183/13993003.00597-2020 
[3] Wang, L.P., Shang, J., Qi, C.L., et al. (2020) Prevention and Control Mechanism and Measures of Nosocomial Infection in a Medical Institution under the Normalization of COVID-19. Chinese Journal of Nosocomiology, 30, 3692-3696

[4] Du, C.L., Wu, Y.Q. and Yang, X.L. (2010) Hospital Response and Disposal Strategies in Public Health Emergencies. Chinese Hospital Management, 30, 59-60

[5] Liu, Z.D., Jin, D.P. and Chen, Z.H. (2003) Hospital Infection Management. Military Medical Science Press, Beijing, China, 3-459.

[6] Chen, L. and Zhang, G.Q. (2020) Intervention of Psychological Problems of Medical Staff under COVID-19 and Ice-Breaking Measures. Medicine and Philosophy, 41, 44-48.

[7] Fu, Q. (2020) Practice of Infection Prevention and Control in Medical Institutions Based on Covid-19. Chinese Journal of Nosocomiology, 30, 1121-1124.

[8] Du, M.M., Yao, H.W. and Bai, Y.L. (2020) Prevention and Control Strategies of Infection in Large General Hospitals during the Epidemic Period of COVID-19. Chinese Journal of Nosocomiology, 30, 1131-1134.

[9] Morton, P.G. (2014) Thanks for the Gift of Reviewers. Journal of professional nursing: Official Journal of the American Association of Colleges of Nursing, 30, 1-2. https://doi.org/10.1016/j.profnurs.2013.12.002

[10] Cao, H.M., Han, G.S., Gu, H., et al. (2020) Construction of Emergency Management System for Hospital Public Health Emergencies Based on Prevention and Control of COVID-19. Chinese Hospital Management, 40, 11-14.

[11] Yuan, L., Liu, F.X., Yin, R., et al. (2020) Practice and Thinking of Epidemic Prevention and Control in Designated Hospitals in COVID-19 from the Perspective of Crisis Management. Modern Hospital, 20, 1097-1099.

[12] Cheng, J., Yang, X. and Chen, Y.J. (2020) Study on Nursing Experience of Intelligent Disinfection Robot in Isolation Ward of COVID-19. Science and Technology and Innovation, 13, 28-31.

[13] WHO (2020) COVID-19 Weekly Epidemiological Update. http://www.who.int/docs/default-source/coronaviruse/situation-reports/20201222 weekly_epi_update_19.pdf?sfvrsn=9ba3df9a_5\&download=true

[14] Han, L., Zhu, S.J. and Wei, H. (2003) The Role of Hospital Infection Management in Dealing with Public Health Emergencies. Chinese Journal of Nosocomiology, 13, 1001-1004.

[15] Wang, D., Hu, B., Hu, C., et al. (2020) Clinical Characteristics of 138 Hospitalized Patients with 2019 Novel Coronavirus-Infected Pneumonia in Wuhan, China. JAMA, 323, 1061-1069. https://doi.org/10.1001/jama.2020.1585 